\section{Severe hypothyroidism after thalidomide treatment}

\author{
Nicola de Savary MAMB ${ }^{1}$ \\ Richard Lee FRCP FRCPath ${ }^{2}$ Bijay Vaidya PhD MRCP ${ }^{1}$
}

J R Soc Med 2004;97:443

An important but little recognized side-effect of thalidomide is hypothyroidism.

\section{CASE HISTORY}

A man aged 49 reported lethargy and back pain and was found to have multiple myeloma. The treatment began with radiotherapy to the spine and repeated courses of vincristine, doxorubicin and dexamethasone. Then, on reaching plateau phase, he underwent autologous stem-cell transplantation following high-dose melphalan. This led to complete remission of the myeloma.

As a part of a clinical trial investigating thalidomide as maintenance therapy for multiple myeloma, he was randomized to receive this drug, initially at $50 \mathrm{mg} /$ day. The dose was increased to $100 \mathrm{mg} /$ day after two weeks and to $200 \mathrm{mg} /$ day after eight weeks. Three months after starting thalidomide, he reported increasing lethargy, constipation and cold intolerance. He had slow-relaxing ankle jerks. There was no goitre. Thyroid function tests revealed gross biochemical hypothyroidism, with thyrotropin $(\mathrm{TSH})>100 \mathrm{mu} / \mathrm{L}$ (normal range $0.25-5.5$ ) and free T4 (FT4) $1.8 \mathrm{pmol} / \mathrm{L}$ (9.7-25.7). Thyroid peroxidase (TPO) antibodies were present. Two days before starting thalidomide his thyroid function had been essentially normal (TSH $0.21 \mathrm{mu} / \mathrm{L}, \mathrm{FT} 423.4 \mathrm{pmol} / \mathrm{L}$ ), and there was no family history of thyroid disease. After the diagnosis of hypothyroidism he was taken off thalidomide and started on thyroxine, with resolution of his symptoms. On thyroxine $125 \mu \mathrm{g}$ daily he remained euthyroid and his multiple myeloma was still in remission eighteen months from diagnosis. The UK Committee on Safety of Medicines say that they received no other reports of thalidomide-induced hypothyroidism between 1966 and 2003.

\section{COMMENT}

Thalidomide was originally marketed in 1956 as a sedative and was withdrawn 5 years later because of teratogenicity. Recently, however, the discovery of its immunomodulatory,

Departments of ${ }^{1}$ Endocrinology and ${ }^{2}$ Haematology, Royal Devon \& Exeter Hospital, Exeter EX2 5DW, UK

Correspondence to: Dr B Vaidya

E-mail: bvaidya@hgmp.mrc.ac.uk anti-inflammatory and anti-angiogenic properties has led to other uses. ${ }^{1}$ One of the more common is the treatment of high-risk refractory multiple myeloma. Others are Behçet's disease, leprosy, pyoderma gangrenosum, HIV infection, graft-versus-host disease, solid organ malignancies, sarcoidosis, Crohn's disease, rheumatoid arthritis and systemic lupus erythematosus. Common side-effects are somnolence, fatigue, constipation, bradycardia, rash and peripheral neuropathy. ${ }^{1}$

Three cases of thalidomide-induced hypothyroidism were reported during the early 1960s..$^{2-4}$ In 2002, Badros and colleagues reported a patient who developed severe hypothyroidism about three months after starting thalidomide for multiple myeloma. ${ }^{5}$ Furthermore, they found that $14 \%$ of patients on thalidomide treatment were subclinically hypothyroid (TSH $>10 \mathrm{mu} / \mathrm{L}$ ) at three months, and suggested that thyroid dysfunction might contribute to some of the known side-effects of the drug, such as fatigue, constipation and bradycardia.

The pathogenesis of thalidomide-induced hypothyroidism is unknown. One possibility is that the drug inhibits secretion of thyroid hormones by a direct action on thyrocytes, ${ }^{6}$ but a more plausible alternative hypothesis is the induction of autoimmune damage to the thyroid gland through its immunomodulatory actions. Thalidomide modulates the production of several cytokines (including tumour necrosis factor- $\alpha$, interferon- $\gamma$, interleukin- 2 , interleukin- 4 , interleukin-5, interleukin-6, interleukin-10 and interleukin-12), alters the ratio of circulating helper (CD4+) T-cells to suppressor $(\mathrm{CD} 8+) \mathrm{T}$-cells, and functions as a potent costimulator of T-cells in vitro, which may trigger an autoimmune response. ${ }^{1,5}$ The finding of TPO antibodies in our patient supports the autoimmune pathogenesis; the status of thyroid autoantibodies in previously reported cases is not known. We do not know whether our patient had TPO antibodies before starting thalidomide.

With the increasing use of thalidomide in diverse medical conditions, this side-effect is likely to be encountered more in the future. We recommend checking thyroid function and TPO antibodies before starting thalidomide, and monitoring thyroid function periodically during treatment.

\section{REFERENCES}

1 Calabrese L, Fleischer AL. Thalidomide: current and potential clinical applications. Am J Med 2000;108:487-95

2 Alexander IRW. Acute myxoedema. BMJ 1961;ii:1434

3 Lillicrap DA. Myxoedema after thalidomide (Distaval). BMJ 1962;i:477

4 Simpson JA. Myxoedema after thalidomide. BMJ 1962;i:55

5 Badros AZ, Siegel E, Bodenner D, et al. Hypothyroidism in patients with multiple myeloma following treatment with thalidomide. Am J Med 2002;112:412-13

6 Somers GF. Pharmacological properties of thalidomide, a new sedative hypnotic drug. Br J Pharmacol 1960;15:111-16 